\title{
Erysipelothrix rhusiopathiae: An Important Cause of Bacterial Disease in Farmed Pigs and an Occupational Pathogen of Humans
}

\section{Margaret Spiteri ${ }^{1}$ and Andrew W. Taylor-Robinson ${ }^{2}$}

${ }^{1}$ School of Health, Medical \& Applied Sciences, Central Queensland University, Rockhampton, QLD, Australia

${ }^{2}$ School of Health, Medical \& Applied Sciences, Central Queensland University, Brisbane, QLD, Australia

\begin{abstract}
Erysipelothrix rhusiopathiae is a Gram-positive bacillus that is found ubiquitously in the environment. It is known to colonise a wide range of animals, most commonly farmed pigs, turkeys and hens, in which it can cause pathology. The disease swine erysipelas affects growing pigs, significantly impacting pork production, and so its early detection and control is economically important. Infection of humans with E. rhusiopathiae is primarily associated with occupational exposure and most often results in a localised skin infection called erysipeloid. More severe infections can cause serious illness and even death in both animals and humans. The accurate identification of E. rhusiopathiae is often dependent on the inclusion in the clinical history of relevant information regarding exposure. Since the bacterium is often mistaken for other species of Grampositive bacillus this ensures that appropriate differential tests are required. There are confirmed cases of $E$. rhusiopathiae infection in individuals with no reported animal exposure so it is possible that there are other modes of transmission. Regarding treatment regimens, E. rhusiopathiae is intrinsically resistant to vancomycin but susceptible to penicillins and carbapenems.
\end{abstract}

Publication History:

Received: September 23, 2018

Accepted: November 08, 2018

Published: November 10, 2018

\section{Keywords:}

Erysipelothrix rhusiopathiae, Swine erysipelas, Septicaemia, Endocarditis, Erysipeloid

\section{Introduction}

Erysipelothrix rhusiopathiae, formally known as Erysipelas insidiosa, is a Gram-positive bacillus that commonly invades and sometimes causes disease in animals, most often farmed pigs, turkeys and chickens, but also sheep, cattle, horses, rodents, wild birds, fish, shellfish and reptiles [1-9]. Humans are infected via zoonotic transmission, which occurs most frequently in those with occupations that involve regular contact with colonised animals, such as farmers, butchers, fish handlers, abattoir workers and livestock veterinarians [1-3,5,7-13]. E. rhusiopathiae is able to infect a broad range of host species, which often act as healthy carriers. Together with the bacterium's ability to survive for weeks to months in soil or dung, this has resulted in a remarkably wide global geographical distribution of E. rhusiopathiae $[1,7,11,12]$.

\section{Presentation and Transmission in Pigs}

E. rhusiopathiae is the causative agent of swine erysipelas, a disease that is described mainly in growing pigs and which thus can have a major negative economic impact on the pig farming industry for production of pork, ham and bacon $[1,3,6-8,12]$. Swine erysipelas may present with any of four syndromes: acute septicaemia; endocarditis; subcutaneous rhomboid urticaria; and arthritis leading to lameness $[1,3,12]$. Acute erysipelas presents with general signs of septicaemia, usually within 24 hours of a pig's exposure to the pathogen, often with skin erythema, petechiae and necrosis [3]; if untreated, it can cause fever and sudden death due to heart failure. Surviving pigs are likely to be condemned at slaughter. In addition, abortions in pregnant sows and infertility in boars may occur. The chronic manifestation of subcutaneous erysipelas starts 2-3 days after exposure, occurring as cutaneous lesions that are frequently diamond-shaped or appear as hives. While lesions typically reduce in severity and disappear in a few days they may continue to spread and intensify, with the possibility of ensuing skin necrosis and/or death [3]. Endocarditis and arthritis may follow previous infection with acute septicaemia or subcutaneous disease [3].

The organism is transmitted not just by swine in a diseased state since E. rhusiopathiae may be detected in the tonsils and lymphoid tissue of up to half of healthy pigs. These may thus act as reservoirs of infection from which the bacterium is passed to other animals in the local proximity $[11,12]$. For this reason management practices have been developed to minimise infection rates, including vaccination, keeping herds closed to prevent the introduction of any potentially infected animals, and the inclusion of antibiotics in commercial livestock feed $[3,12]$. Swine erysipelas represents one of the most common clinical problems encountered on commercial pig farms, particularly in open-pen straw yard systems. It is also encountered in pigs kept in small populations such as smallholdings, hobby farms and specialist pedigree small herds. Carriers shed E. rhusiopathiae in faeces, urine, saliva and nasal secretions, leading to contamination of soil, food and water, thereby facilitating indirect transmission of the pathogen [3].

\section{Presentation and Transmission in Humans}

Infection in humans is usually established via bacillus entry through broken skin, presenting as localised cutaneous cellulitis called erysipeloid $[1-5,7,8,12]$. This condition is characterised by swelling and redness of the infected areas, most commonly the hands due to the typically occupational nature of transmission [10]. The often severely painful lesions spread outwards as the central area fades in colour, but the condition frequently resolves within a month without treatment [3]. A diffuse cutaneous infection, infectious endocarditis and sepsis are less common presentations of E. rhusiopathiae colonisation of humans, each of which requires treatment and often recurs [1$5,7,8,10,12]$. Rare reports of human infection with E. rhusiopathiae in patients with no recent history of animal contact suggest that transmission through contaminated soil or other environmental

"Corresponding Author: Prof. Andrew W. Taylor-Robinson, School of Health, Medical \& Applied Sciences, Central Queensland University, 160 Ann Street, Brisbane, QLD 4000, Australia, Tel: +61 73295 1185; E-mail: a.taylor-robinson@cqu.edu.au

Citation: Spiteri M, Taylor-Robinson AW (2018) Erysipelothrix rhusiopathiae: An Important Cause of Bacterial Disease in Farmed Pigs and an Occupational Pathogen of Humans. Int J Clin Med Microbiol 3: 134. doi: https://doi. org/10.15344/2456-4028/2018/134

Copyright: (C) 2018 Spiteri et al. This is an open-access article distributed under the terms of the Creative Commons Attribution License, which permits unrestricted use, distribution, and reproduction in any medium, provided the original author and source are credited. 
Citation: Spiteri M, Taylor-Robinson AW (2018) Erysipelothrix rhusiopathiae: An Important Cause of Bacterial Disease in Farmed Pigs and an Occupational Pathogen of Humans. Int J Clin Med Microbiol 3: 134. doi: https://doi.org/10.15344/2456-4028/2018/134

Page 2 of 3

sources may be possible, although human-to-human transmission has never been described $[1,2,11]$. Other rare forms of human infection with E. rhusiopathiae have been documented, including pneumonia in an immunocompetent patient [4], osteomyelitis two decades after traumatic inoculation with E. rhusiopathiae by a cow's horn [13], endocarditis with bacteraemia preceding osteomyelitis of the spine [2], and osseous necrosis of a thumb resulting in fatal endocarditis, intracranial abscess and chronic arthritis [1].

\section{Laboratory Identification}

E. rhusiopathiae is a straight or slightly curved, thin, non-motile, catalase and oxidase negative, non-acid-fast, non-spore-forming rod. The bacterium is facultatively anaerobic, possesses a capsule and is found intracellularly in infected organisms $[1-4,7,11,12]$. Despite being classified as Gram-positive E. rhusiopathiae is easily discoloured and may be mistaken as Gram-negative if due care is not taken with microscopical examination of stained preparations $[1,3,12]$. Colonies of E. rhusiopathiae are commonly but not always alpha-haemolytic on blood agar, and produce hydrogen sulphide on triple sugar iron medium [1-3]. The bacterium is negative for methyl red, indole, esculin, nitrate reduction, the Voges-Proskauer test to detect acetoin, and liquefaction of gelatine $[1,3]$. In addition, it produces acid from glucose, fructose, galactose and lactose but not from maltose, zylose and mannitol, and it does not ferment sucrose $[1,3]$. Direct and indirect fluorescent antibody tests can be used to identify E. rhusiopathiae, as well as PCR methods, while rapid identification can be achieved using the well-established commercial API Coryne System (bioMèrieux, France) for microorganism identification to the species level [3].

Several microbiological media that are selective for the growth of Erysipelothrix have been developed. These include: Erysipelothrix selective broth, which contains serum, tryptose, kanamycin, neomycin and vancomycin; modified blood azide medium, which contains sodium azide and horse blood or serum; Bohm's medium, which contains sodium azide, kanamycin, phenol and water blue; and Shimoji's selective enrichment broth, which contains tryptic soy broth, Tween 80 , Tris-aminomethane, crystal violet and sodium azide $[1,3]$. Packer's medium containing sodium azide and crystal violet is useful for grossly contaminated specimens $[1,3]$.

Colony morphology has been described as clear, circular and very small $\left(0.1-0.5 \mathrm{~mm}\right.$ after 24 hours incubation at $37^{\circ} \mathrm{C}$, or 0.5 $1.5 \mathrm{~mm}$ after 48 hours incubation at the same temperature) $[1,3]$. E. rhusiopathiae is dimorphic and can be distinguished on solid media as well as under the optical microscope. Smooth colonies appear bluish, transparent and convex, consisting of small, thin, slightly curved rods with rounded ends. Rough colonies are larger with a flat but irregular surface and crenate edges, and consist of long filaments, often seen in chains $[1,3,5]$. It has been suggested that colony morphology may change between the two manifestations dependent upon variations in $\mathrm{pH}$ and temperature $[1,3]$.

E. rhusiopathiae is often confused morphologically with other Gram-positive, non-spore-forming bacilli such as Brochothrix, Corynebacterium, Kurthia, Lactobacillus and Listeria [1,3]. For this reason E. rhusiopathiae infections in humans are likely to be underreported. Hence, care should be taken to ensure a proper history of a patient's contact with animals is included in clinical notes if there is any suggestion that this bacterium may be the causative agent. This will enable the appropriate microbiological tests to be applied and thus increase the prospects of the correct diagnosis.

\section{Serotypes and Virulence Factors}

A number of different serotypes of E. rhusiopathiae have been identified in animals, with particular serotypes associated with distinct disease states in various host species. Most cases of swine erysipelas are caused by either serotype $1 \mathrm{a}, 1 \mathrm{~b}$ or $2[3,10]$. Very little information is available regarding the serotypes implicated in human cases but one study identified serotype 3 in a patient with endocarditis caused by E. rhusiopathiae [10]. Another serotype isolated from a different endocarditis patient in the same study was untypeable, suggesting the presence of an unknown, possibly new, serotype that is yet to be classified [10].

The virulence of $E$. rhusiopathiae varies with serotype $[3,8,10]$. The mechanism(s) of pathogenicity are not yet well understood, although no toxin has been characterised and several potential virulence factors have been identified. These include neuraminidase, an enzyme that cleaves sialic acids in glycoproteins, glycolipids and oligo and polysaccharides, thereby possibly serving to disrupt host cell functions as well as bacterial cell capsular antigens, the surface protective antigen and haemolysin $[3,8]$

\section{Prevention, Treatment and Resistance}

Containment of infected animals and known carriers, as well as removal or regular cleaning and disinfecting of contaminated materials, have been demonstrated as effective control measures against the spread of E. rhusiopathiae infection in both animals and humans [3]. Commercial vaccines typically incorporate serotypes 1 and 2. Immunisation of livestock is recommended and generally practiced by large farming enterprises, although as yet a human vaccine has not been developed successfully [3,12]. For those people working in occupations that carry an intrinsically high risk of E. rhusiopathiae infection, awareness is important. The use of protective gloves, good hygiene practices and the prompt treatment of any injuries that cause skin to be broken, no matter how small, are all essential for preventing (spread of) infection [3].

E. rhusiopathiae has been found to be sensitive to the penicillin and carbapenem groups of antibiotics but it is resistant to vancomycin [14]. Oral doses of penicillin are sufficient to resolve most erysipeloid cases but intravenous penicillin is recommended for more serious infections [3].

\section{Conclusion}

E. rhusiopathiae is a notable pathogen of a wide range of animals that is ubiquitous in nature. Colonisation of humans is usually associated with occupational exposure to infected animals, especially pigs, but infection has been reported for patients with no known animal contact. Training in best practice handling of live and slaughtered animals will help to reduce the risk of zoonotic transmission to people performing their jobs. E. rhusiopathiae can be mistaken by microscopical examination for other morphologically similar Grampositive bacillus species, some of which would be seen more routinely by a diagnostic microbiology laboratory. Hence, further specific biochemical tests are recommended in order to make an accurate diagnosis and thus to prescribe the appropriate treatment. Further research is required to better understand the pathogenicity and virulence of different serotypes of E. rhusiopathiae. 
Citation: Spiteri M, Taylor-Robinson AW (2018) Erysipelothrix rhusiopathiae: An Important Cause of Bacterial Disease in Farmed Pigs and an Occupational Pathogen of Humans. Int J Clin Med Microbiol 3: 134. doi: https://doi.org/10.15344/2456-4028/2018/134

Page 3 of 3

\section{Author Contributions}

Both authors have made substantial contributions to the conception of the article, contributed significantly to writing the manuscript, revised it critically for important intellectual content, approved its final version and agreed to its submission.

\section{Competing Interests}

The authors declare that they have no competing interests.

\section{References}

1. Reboli AC, Farrar WE (1989) Erysipelothrix rhusiopathiae: an occupational pathogen. Clin Microbiol Rev 2: 354-359.

2. Romney $M$, Cheung $S$, Montessori V (2001) Erysipelothrix rhusiopathiae endocarditis and presumed osteomyelitis. Can J Infect Dis 12: 254-256.

3. Wang Q, Chang BJ, Riley TV. (2010) Erysipelothrix rhusiopathiae. Vet Microbiol 140: 405-417.

4. Meric M, Ozcan SK (2012) Erysipelothrix rhusiopathiae pneumonia in an immunocompetent patient. J Med Microbiol 61: 450-451.

5. Tille P (2018) Bailey \& Scott's Diagnostic Microbiology (14th edition), St. Louis: Elsevier, USA.

6. Zou Y, Zhu X, Muhammad HM, Jiang P, Li Y, et al. (2015) Characterization of Erysipelothrix rhusiopathiae strains isolated from acute swine erysipelas outbreaks in Eastern China. J Vet Med Sci 77: 653-660.

7. Forde T, Biek R, Zadoks R, Workentine ML, De Buck J, et al. (2016) Genomic analysis of the multi-host pathogen Erysipelothrix rhusiopathiae reveals extensive recombination as well as the existence of three generalist clades with wide geographic distribution. BMC Genomics 17: 461.

8. Li Y, Zou Y, Xia Y, Bai J, Wang X, et al. (2016) Proteomic and transcriptomic analyses of swine pathogen Erysipelothrix rhusiopathiae reveal virulence repertoire. PLoS One 11: e0159462.

9. Principe L, Bracco S, Mauri C, Tonolo S, Pini B, et al. (2016) Erysipelothrix rhusiopathiae bacteremia without endocarditis: rapid identification from positive blood culture by MALDI-TOF mass spectrometry. A case report and literature review. Infect Dis Rep 8: 6368.

10. Harada K, Amano K, Akimoto S, Yamamoto K, Yamamoto Y, et al. (2011) Serological and pathogenic characterization of Erysipelothrix rhusiopathiae isolates from two human cases of endocarditis in Japan. New Microbiol 34 409-412.

11. Boyd AS, Ritchie C, Fenton JS (2014) Cutaneous Erysipelothrix rhusiopathiae (erysipeloid) infection in an immunocompromised child. Pediatr Dermatol 31: 232-235.

12. Clark AE (2015) The occupational opportunist: an update on Erysipelothrix rhusiopathiae infection, disease pathogenesis, and microbiology. Clin Microbiol Newsl 37: 143-151.

13. Denes E, Camilleri Y, Fiorenza F, Martin C (2015) First case of osteomyelitis due to Erysipelothrix rhusiopathiae: pubic osteomyelitis in a gored farmer. Int J Infect Dis 30: 133-134. 